\section{ORIGINAL RESEARCH}

\author{
Z.I. Hasiloglu \\ S. Albayram \\ H. Selcuk \\ E. Ceyhan \\ S. Delil \\ B. Arkan \\ L. Baskoy
}

\title{
Cerebral Microhemorrhages Detected by Susceptibility-Weighted Imaging in Amateur Boxers
}

BACKGROUND AND PURPOSE: SWI is a new technique for evaluating diffuse axonal injury associated with punctate hemorrhages. The aim of our study was to determine the prevalence of cerebral microhemorrhages in amateur boxers compared with nonboxers by using SWI and to evaluate the sensitivity of SWI compared with T2 FSE and T2* GE sequences.

MATERIALS AND METHODS: We performed cranial MR imaging with a $1.5 \mathrm{~T}$ scanner in 21 amateur boxers and 21 control subjects. The study protocol included conventional MR images, T2 FSE, T2* GE, and SWI sequences. The proportions of boxers and controls having CSP, DPVS, cerebral atrophy, cerebellar atrophy, ventricular dilation, PSWMD, and microhemorrhages were computed and were compared by using the $\chi^{2}$ test of proportions. The relationship between microhemorrhages and boxing-related covariates was assessed by using the Wilcoxon rank sum test. The association between the categories was tested by using the Fisher exact test.

RESULTS: Using SWI, microhemorrhages were found in 2 (9.52\%) of 21 boxers. The microhemorrhages were not visible on T2 FSE or T2* GE images. The proportion of subjects with microhemorrhages did not differ significantly between the boxers and control subjects $\left(\chi^{2}=0.525, d f=1, P=\right.$ .4688). The prevalence of CSP and DPVS was significantly higher in the boxers than in the control subjects.

CONCLUSIONS: More microhemorrhages were detected in amateur boxers than in controls, but this difference was not statistically significant.

\begin{abstract}
ABBREVIATIONS: $A=$ active boxer; $\mathrm{CSP}=$ cavum septum pellucidum; $\mathrm{DAI}=$ diffuse axonal injury; DPVS = dilated perivascular space; $\mathrm{GE}=$ gradient-echo; $\mathrm{KO}=$ knockout; $\mathrm{MH}=$ microhemorrhages; MMSE = Mini-Mental State Examination; PSWMD = periventricular and subcortical white matter disease; $R=$ retired boxer; $S E=$ spin-echo; $S W I=$ susceptibility-weighted imaging; $T 2$ $\mathrm{FSE}=\mathrm{T} 2$-weighted fast spin-echo; $\mathrm{T} 2{ }^{*} \mathrm{GE}=\mathrm{T} 2{ }^{*}$-weighted $\mathrm{GE} ; \mathrm{TBI}=$ traumatic brain injury
\end{abstract}

$\mathbf{B}^{\mathrm{r}}$ rain injuries from repetitive head blows have been reported in the boxing population. ${ }^{1} \mathrm{TBI}$ as a result of boxing is usually termed "chronic traumatic encephalopathy," which represents a cumulative process of repetitive head blows in contact sports. ${ }^{2}$ Sudden acceleration of the head caused by the impact of a blow during a boxing fight may result in a cerebral contusion and DAI from axonal stretching. DAI can be detected by MR imaging as small round or ovoid lesions, representing multifocal punctate hemorrhages. ${ }^{3} \mathrm{~A} T 2^{\star} \mathrm{GE}$ sequence is more reliable for detecting the size, number, volume, and distribution of microhemorrhagic lesions than other more classic MR imaging sequences. ${ }^{4}$ Nevertheless, the evidence for TBI in amateur boxers is controversial. ${ }^{5-7}$

SWI is a new technique for the evaluation of DAI associated with punctate hemorrhages in brain parenchyma. The aim of our study was to determine the prevalence of cerebral microhemorrhages in amateur boxers compared with nonboxers by using SWI and to evaluate the sensitivity of SWI compared

Received May 10, 2010; accepted after revision June 11.

From the Department of Radiology (Z.I.H., S.A.), Division of Neuroradiology, and Department of Neurology (S.D.), Istanbul University, Cerrahpasa Medical School (B.A., L.B.), Istanbul, Turkey; Department of Radiology (H.S.), Bakirkoy Dr. Sadi Konuk Training and Research Hospital, Istanbul, Turkey; and Department of Mathematics (E.C.), Koc University, Istanbul, Turkey.

Please address correspondence to Sait Albayram, MD, Cerrahpasa Medical School, Department of Radiology, Division of Neuroradiology, KMP Istanbul, Turkey 34300; e-mail: salbayram@hotmail.com

DOI 10.3174/ajnr.A2250 with T2 FSE and $\mathrm{T} 2{ }^{\star} \mathrm{GE}$ sequences for assigning the number of microhemorrhages in amateur boxers. We hypothesized that the number of microhemorrhages is more accurately evaluated with SWI. The presence of findings that can be developed secondary to chronic trauma such as CSP, DPVS, cerebral atrophy, cerebellar atrophy, ventricular dilation, PSWMD, and associations of those findings with microhemorrhages were also investigated with conventional MR images.

\section{Materials and Methods}

We studied 21 male amateur classic boxers ranging in age from 19 to 59 years (mean, 42.95 years) and 21 healthy nonboxing male volunteers, who also ranged in age from 19 to 59 years. The mean duration of boxing was 12.9 years (range, 5-35 years). The total number of fights ranged from 15 to 200 , and the weight divisions were between 54 and $107 \mathrm{~kg}$. Eight subjects were active boxers, and 13 were retired. Most subjects boxed before head guards were obligatory and, therefore, never used a head guard. Only 8 boxers fought with a head guard in all of their matches. Ten boxers had been knocked out in the past, and the total number of knockouts in each boxer ranged from 0 to 8 . Nine of the 10 lost consciousness for 5 seconds to 30 minutes. One boxer never lost consciousness, despite having been knocked out.

Boxers and control subjects with neurologic or other diseases involving the central nervous system, head trauma, epilepsy, metabolic disorders, and perinatal asphyxia, were excluded from this study. Drug abusers were also excluded. Hypertensive subjects were excluded from the study, and both boxers and control group were nor- 


\begin{tabular}{|c|c|c|c|c|c|c|c|c|c|c|c|c|}
\hline \multicolumn{13}{|c|}{ History and MR imaging findings of the boxers } \\
\hline Case & Age (yr) & $\begin{array}{c}\text { Boxing } \\
\text { Duration (year) }\end{array}$ & $\begin{array}{l}\text { Total No. } \\
\text { of Fights }\end{array}$ & $\begin{array}{c}\text { Total No. } \\
\text { of KOs }\end{array}$ & $\begin{array}{l}\text { Working } \\
\text { Situation }\end{array}$ & CSP & DPVS & $\begin{array}{l}\text { Cerebral } \\
\text { Atrophy }\end{array}$ & $\begin{array}{c}\text { Cerebellar } \\
\text { Atrophy }\end{array}$ & $\begin{array}{l}\text { Ventricular } \\
\text { Dilation }\end{array}$ & PSWMD & $\mathrm{MH}$ \\
\hline 1 & 46 & 17 & 170 & 1 & $A$ & + & + & - & - & - & - & $\overline{-}$ \\
\hline 2 & 53 & 12 & 150 & 0 & $A$ & - & + & - & - & - & + & - \\
\hline 3 & 58 & 35 & 180 & 0 & $\mathrm{R}$ & - & + & - & - & - & - & - \\
\hline 4 & 56 & 11 & 100 & 0 & $\mathrm{R}$ & + & + & - & - & - & - & - \\
\hline 5 & 59 & 15 & 190 & 8 & $\mathrm{R}$ & + & + & + & + & + & + & - \\
\hline 6 & 50 & 8 & 50 & 0 & $\mathrm{R}$ & - & + & - & - & + & + & - \\
\hline 7 & 19 & 6 & 50 & 1 & $A$ & - & + & - & - & - & + & - \\
\hline 8 & 34 & 12 & 100 & 0 & $A$ & - & + & - & - & - & - & - \\
\hline 9 & 30 & 14 & 100 & 0 & A & + & + & - & - & - & - & - \\
\hline 10 & 34 & 6 & 15 & 2 & $A$ & + & + & - & - & - & - & - \\
\hline 11 & 43 & 14 & 140 & 4 & $\mathrm{R}$ & - & + & - & - & + & - & + \\
\hline 12 & 50 & 17 & 200 & 2 & $\mathrm{R}$ & + & + & + & + & + & - & + \\
\hline 13 & 51 & 15 & 100 & 1 & $\mathrm{R}$ & + & + & - & - & + & + & - \\
\hline 14 & 34 & 10 & 60 & 0 & $\mathrm{R}$ & + & - & - & - & - & - & - \\
\hline 15 & 44 & 22 & 200 & 0 & $\mathrm{R}$ & + & + & - & + & - & + & - \\
\hline 16 & 40 & 11 & 115 & 0 & $\mathrm{R}$ & + & + & - & + & - & + & - \\
\hline 17 & 50 & 12 & 40 & 0 & $\mathrm{R}$ & - & + & - & - & - & + & - \\
\hline 18 & 19 & 5 & 40 & 4 & A & - & + & - & - & - & - & - \\
\hline 19 & 28 & 8 & 55 & 0 & A & - & + & - & - & - & - & - \\
\hline 20 & 51 & 10 & 50 & 2 & $\mathrm{R}$ & + & + & - & - & - & - & - \\
\hline 21 & 53 & 11 & 75 & 1 & $\mathrm{R}$ & + & - & - & - & - & - & - \\
\hline
\end{tabular}

Note:-+ indicates present; -, absent.

motensive. Neurologic examinations were performed in subjects with dementia, depression, tremors, nystagmus, speech disorders, or forgetfulness. An MMSE was performed to evaluate the presence of chronic traumatic encephalopathy. The data from patients and control subjects were acquired between January 2009 and February 2010.

MR images were acquired by using a $1.5 \mathrm{~T}$ unit (Magnetom Avanto; Siemens, Erlangen, Germany) with an 8-channel head coil. The MR imaging protocols included transverse T2 FSE imaging (TR/TE, 4820/116 ms; section thickness, $5.5 \mathrm{~mm}$; flip angle, $30^{\circ}$; acquisition matrix, $512 \times 256 \mathrm{~ms}$; and FOV, $230 \mathrm{~mm}$ ); transverse T1-weighted SE imaging (TR/TE, 409/10 ms; section thickness, $5.5 \mathrm{~mm}$; flip angle, $90^{\circ}$; acquisition matrix, $512 \times 256 \mathrm{~ms}$; and FOV, $230 \mathrm{~mm}$ ); transverse fluid-attenuated inversion recovery imaging (TR/TE, 9040/103 ms; section thickness, $5.5 \mathrm{~mm}$; flip angle, 30ªcquisition matrix, $512 \times 256 \mathrm{~ms}$; and FOV, $230 \mathrm{~mm}$ ); coronal T2 FSE imaging (TR/TE, 4190/107 ms; section thickness, $5.5 \mathrm{~mm}$; flip angle, $30^{\circ}$; acquisition matrix, $512 \times 256 \mathrm{~ms}$; and FOV, $\left.230 \mathrm{~mm}\right)$; sagittal T1-weighted SE imaging (TR/TE, 408/10 ms; section thickness, $5 \mathrm{~mm}$; flip angle, $90^{\circ}$; acquisition matrix, $512 \times 256 \mathrm{~ms}$; and FOV, $230 \mathrm{~mm}$ ); transverse $\mathrm{T} 2{ }^{\star} \mathrm{GE}$ imaging (TR/TE, 660/24 ms; section thickness, $5.5 \mathrm{~mm}$; flip angle, $20^{\circ}$; acquisition matrix, $512 \times 256 \mathrm{~ms}$; and FOV, $230 \mathrm{~mm}$ ); and a transverse SWI sequence (TR/TE, 49/40 ms; section thickness, $2 \mathrm{~mm}$; flip angle, $20^{\circ}$; bandwidth, $80 \mathrm{~Hz} /$ pixel; acquisition matrix, $512 \times 256 \mathrm{~ms}$; and FOV, $230 \mathrm{~mm}$ ).

All images were acquired in the same axial plane. Subsequently, the SWI sequences were reconstructed by using a minimumintensity-projection technique, to obtain images with section number, thickness, and position similar to those of T2 FSE and T2 $2^{\star} \mathrm{GE}$ sequences. MR imaging data were made anonymous before evaluation. The study protocol was approved by our institutional review board, and informed consent was obtained from each subject.

Two experienced neuroradiologists independently reviewed the MR images. Conventional MR imaging, $\mathrm{T} 2{ }^{\star} \mathrm{GE}$, and SWI images were evaluated independently in all 42 subjects (21 boxers and 21 agematched controls). Various conditions such as CSP, DPVS, cerebral atrophy, cerebellar atrophy, ventricular dilation, PSWMD, and microhemorrhages were evaluated on conventional MR images of boxers and control subjects, who were categorized according to the presence (yes) or absence (no) of these conditions. Microhemorrhages were defined as small round lesions with a homogeneous very low signal intensity on $\mathrm{T} 2{ }^{\star} \mathrm{GE}$ or SWI, without continuity with surrounding vascular structures.

The proportions of boxers and controls having CSP, DPVS, cerebral atrophy, cerebellar atrophy, ventricular dilation, PSWMD, and microhemorrhages were computed and were compared by using the $\chi^{2}$ test of proportions. The boxer data were analyzed according to duration of boxing, working situation, total number of fights, and total number of knockouts, and according to CSP, DPVS, cerebral atrophy, cerebellar atrophy, ventricular dilation, PSWMD, and microhemorrhages with respect to boxing-related covariates such as duration of boxing in years, total number of fights, and total number of knockouts. Our main goal was to assess the relationship between microhemorrhages and these boxing-related covariates. The Wilcoxon rank sum test was used to test the significance of the differences in covariates between the groups of boxers (eg, the difference in the number of fights between boxers with CSP and boxers without CSP). Finally, the association between the categories (eg, whether microhemorrhage, CSP, and DPVS tended to occur together in boxers) was tested by using the Fisher exact test for contingency tables.

\section{Results}

The histories and MR imaging findings in boxers are summarized in the Table. MMSE revealed dementia in 1 case (subject $5)$. In this case, a chronic subdural hematoma was detected, but a microhemorrhagic focus could not be detected with SWI. On conventional MR imaging, 19 boxers (90\%) had DPVS; 12 (57\%), CSP; 8 (38\%), PSWMD; 5 (24\%), ventricular dilation; 4 (19\%), cerebellar atrophy; 2 (9.5\%), cerebral and cerebellar atrophy; and 1 (4.7\%), chronic subdural hemorrhage. On SWI, microhemorrhages, appearing as hypointense foci, were found in $2(9.52 \%)$ of the 21 boxers (subjects 11 and 12) and were located exclusively in the brain stem. 
The microhemorrhages were not visible on conventional $\mathrm{MR}$ imaging or $\mathrm{T} 2{ }^{\star} \mathrm{GE}$ images.

In subject 12 , a millimetrically sized second hypointense focus was also seen in the right frontal convexity. This focus was observed hypointensely in $\mathrm{T} 2{ }^{\star} \mathrm{GE}$ and SWI magnitude images and was evident hyperintensely in SWI phase images. It was thought that this lesion might have been a calcification. In another case (subject 3), a cavernous malformation was incidentally found and easily detected by using conventional MR imaging, $\mathrm{T} 2{ }^{\star} \mathrm{GE}$, and SWI.

The prevalence of microhemorrhages did not differ significantly between the boxers and the control subjects $\left(\chi^{2}=\right.$ $0.525, d f=1, P=.4688)$. On the other hand, the prevalence of CSP was significantly higher in the boxers than in the control subjects $\left(\chi^{2}=14.1167, d f=1, P<.0001\right)$, suggesting a significant association between boxing and CSP. The prevalence of DPVS was also significantly higher in the boxers than in the control subjects $\left(\chi^{2}=4.434, d f=1, P=.0176\right)$, suggesting a significant association between boxing and DPVS. However, the prevalences of cerebral atrophy, cerebellar atrophy, ventricular dilation, and PSWMD did not differ significantly between the boxers and the control subjects $(P<.05$ for all).

There was no significant association between microhemorrhage and DPVS, CSP, cerebral atrophy, cerebellar atrophy or ventricular dilation (Fisher exact test, $P>.05$ for all). Boxingrelated covariates (duration of boxing, number of fights, and number of knockouts) did not differ significantly between boxers with and without microhemorrhages on the basis of the Wilcoxon rank sum test $(P>.05)$.

\section{Discussion}

The neuropathologic findings in TBI are tearing of the septum pellucidum with thalamic gliosis, atrophy and gliosis of the inferior cerebellar folia, and loss of pigmented neurons in the substantia nigra. ${ }^{6}$ Cerebral and cerebellar atrophy, ventricular dilation, posttraumatic cysts, cavum septum pellucidum, cerebral contusion, intraparenchymal and extra-axial hematomas, axonal tearing, and white matter microhemorrhages have been seen in boxing trauma. ${ }^{1,8-11}$ TBI in boxing has been presented as either focal or DAI. ${ }^{12}$ DAI associated with punctuate hemorrhages in the deep subcortical white matter and brain stem is not routinely visible on CT or conventional MR imaging sequences. Detection of the number, size, and location of microhemorrhages may help to explain the clinical findings and severity of brain damage due to repeated head blows. Additionally, the involvement of the brain-stem in DAI is a very important predictor of long-term outcome. ${ }^{4}$

The largest study of CT scans in boxers was that of Jordan et al, ${ }^{11}$ who evaluated 388 active professional boxers. CT findings were normal in 93\% of the boxers and showed "borderline" atrophy in $6 \%$. Boxers with CSP (14\%) were more likely to have cerebral atrophy. One of the earliest studies using MR imaging in boxers was performed by Cabanis et al. ${ }^{13}$ Of the 12 active and 40 retired boxers (13 amateur, 39 professional) examined, only 2 exhibited CSP. Cerebral atrophy was noted in 8 , and the mamillary bodies and optic chiasm were described as small in 30. No correlative clinical data were presented, and given that this study was performed by using a $0.15 \mathrm{~T}$ scanner with 9-mm sections in the early days of MR imaging, technical limitations are likely to have restricted the conclusions drawn from their findings.

Levin et $\mathrm{al}^{14}$ and Jordan and Zimmerman ${ }^{15}$ each studied 9 amateur boxers and reported no abnormal MR imaging findings. Jordan and Zimmerman ${ }^{8}$ subsequently examined 21 active boxers (16 professional) and 1 retired professional boxer. Most of the CT and MR imaging findings were normal, and no clear relationship between boxing exposure and radiologic findings was noted. The authors emphasized the superiority of MR imaging over CT with respect to its usefulness in delineating equivocal CT abnormalities. The most recent systematic study on structural brain abnormalities in amateur boxers as detected by MR imaging was performed in 1992 by Holzgraefe et al, ${ }^{16}$ who found no brain abnormalities and a $0 \%$ prevalence of cerebral microhemorrhages in 13 boxers. However, this study was performed on a $0.5 \mathrm{~T} \mathrm{MR}$ imaging scanner, and images of only 10 -mm thickness were acquired. In postmortem studies of retired boxers, superficial siderosis of the cerebellum has been discovered. ${ }^{17}$ Although this should be detectable by using MR imaging, no case reports exist in which this condition has been noted.

Several recent studies have used newly developed MR imaging techniques to elucidate the pathology in the boxing population. Zhang et $\mathrm{al}^{1}$ performed a study on 24 professional boxers and observed no statistically significant increase in the detected diffusion value, compared with the value in the control group. In another study by Zhang et $\mathrm{al}^{2}{ }^{2}$ normal conventional MR imaging findings were detected in 42 of 49 professional boxers. Nonspecific white matter lesions were detected in the remaining 7. However, a significant difference in diffusion anisotropy measurements was detected between boxers and the control group. In boxers, an increased brain average diffusion constant and decreased diffusion anisotropy values were found in the corpus callosum and internal capsule, and it was emphasized that these findings may be preclinical signs of brain injury in professional boxers. The presence of microhemorrhage was not sought in either study.

A 3T MR imaging study by Hahnel et $\mathrm{al}^{3}$ showed that microhemorrhages were present in $7 \%$ of the subjects. Although the prevalence was higher in 42 amateur boxers than in the control group, the prevalence was not significantly different between boxers ( 3 cases) and controls ( 0 cases). In the present study, microhemorrhages were detected by using 3T MR imaging and GE sequencing, with 1-mm section thickness. In 1 boxer, 2 separate microhemorrhages were detected, whereas 2 other boxers had only 1 lesion each. The microhemorrhages were located exclusively in the corticomedullary junction of the frontal or temporal lobe.

SWI is a full-velocity-compensated high-resolution 3D GE sequence. SWI provides additional clinically useful information that is often complementary to conventional MR imaging sequences and is used in the evaluation of various neurologic disorders, including TBI and coagulopathic or other hemorrhagic disorders. SWI is particularly helpful for the evaluation of DAI, often associated with punctuate hemorrhages in the deep subcortical white matter, which are not routinely visible on CT or conventional MR imaging sequences. It exploits the magnetic susceptibility differences between tissues, resulting in phase differences between regions containing paramagnetic deoxygenated blood products (deoxyhemoglobin, intracellu- 
lar methemoglobin, or hemosiderin) and surrounding tissue. ${ }^{4}$ Studies by Tong et $\mathrm{al}^{18,19}$ and Babikian et $\mathrm{al}^{20}$ have shown that SWI has 3-6 times the sensitivity of conventional T2* GE sequences for detecting the size, number, volume, and distribution of hemorrhagic lesions in DAI. Furthermore, Babikian et al demonstrated that the number and volume of SWI hemorrhagic lesions appeared to correlate with specific neuropsychological deficits.

In the present study, the prevalence of cerebral microhemorrhage was higher in the boxers $(2 / 21 ; 9.5 \%)$ compared with the nonboxer controls $(0 / 21 ; 0 \%)$, but the difference was not statistically significant $\left(\chi^{2}=0.525, d f=1, P=.4688\right)$. The 2 foci of microhemorrhages detected in the boxers could be seen only on SWI images and were not detectable by using T2 FSE or $\mathrm{T} 2{ }^{\star} \mathrm{GE}$. Most interesting, the 2 microhemorrhages were in the brain stem, which is a typical location for DAI and results in axonal stretching. Thus, the usefulness of SWI in the detection of brain stem lesions was recognized. In either SWI or $\mathrm{T} 2{ }^{\star} \mathrm{GE}$, susceptibility artifacts, particularly those close to bone, may hide peripheral lesions.

Our study has some limitations. First, the study was performed on an unsatisfactory number of boxers. Second, although most boxers had trained and fought for a long time, some had not fought recently because they had retired $(n=13$, $61.9 \%$ ). Although SWI is a sensitive imaging technique for the detection of acute-to-subacute hemorrhaging, foci of chronic hemorrhaging, as might have been present in the retired boxers, may not be seen in SWI due to hemorrhage resorption. Third, the prevalence of microhemorrhages in the boxing population, especially in boxers with knockouts, may be higher than that found in our study because most of the boxers $(n=11,52.38 \%)$ in our study had never been knocked out. In other studies, the number who had been knocked out ranged from 1 to 8 .

\section{Conclusions}

SWI is an effective imaging method for the detection of cerebral microhemorrhages, an important form of TBI in boxers. Compared with the control group, the amateur boxers had more microhemorrhages, but this difference was not statistically significant. Imaging should be performed on large study groups of boxers who have boxed professionally for a long time, have been knocked out numerous times, and have developed clinically chronic traumatic encephalopathy, to more clearly understand the efficiency of SWI. By so doing, the importance of DAI and microhemorrhages to the chronic traumatic encephalopathy pathophysiology that develops after boxing could be recognized.

\section{References}

1. Zhang L, Ravdin LD, Relkin N, et al. Increased diffusion in the brain of professional boxers: a preclinical sign of traumatic brain injury? AJNR Am J Neuroradiol 2003;24:52-57

2. Zhang L, Heier LA, Zimmerman RD, et al. Diffusion anisotropy changes in the brains of professional boxers. AJNR Am J Neuroradiol 2006;27:2000-04

3. Hahnel S, Stippich C, Weber I, et al. Prevalence of cerebral microhemorrhages in amateur boxers as detected by $3 \mathrm{~T}$ MR imaging. AJNR Am J Neuroradiol 2008;29:388-91

4. Mittal S, Wu Z, Neelavalli J, et al. Susceptibility-weighted imaging: technical aspects and clinical applications, Part 2. AJNR Am J Neuroradiol 2009; 30:232-52

5. Kemp PM, Houston AS, Macleod MA, et al. Cerebral perfusion and psychometric testing in military amateur boxers and controls. J Neurol Neurosurg Psychiatry 1995;59:368-74

6. Moseley IF. The neuroimaging evidence for chronic brain damage due to boxing. Neuroradiology 2000;42:1-8

7. Loosemore M, Knowles CH, Whyte GP. Amateur boxing and risk of chronic traumatic brain injury: systematic review of observational studies. $B M J$ 2007;335:809

8. Jordan $\mathrm{BD}$, Zimmerman RD. Computerized tomography and magnetic resonance imaging comparison in boxers. JAMA 1990;263:1670-74

9. Roberts AH. Brain Damage in Boxers: A Study of the Prevalence of Traumatic Encephalopathy among Ex-Professional Boxers. London, UK: Pitman Medical; 1969

10. Corsellis JA. Boxing and the brain. BMJ 1989:105-09

11. Jordan BD, Jahre C, Hauser WH, et al. CT of 388 active professional boxers. Radiology 1992;185:509-12

12. Chappell MH, Ulug AM, Zhang L, et al. Distribution of microstructural damage in the brains of professional boxers: a diffusion MRI study. J Magn Reson Imaging 2006;24:537-42

13. Cabanis EA, Perez G, Tamraz JC. Cephalic magnetic resonance imaging of boxers. Acta Radiol 1986;369(suppl):365-66

14. Levin HS, Lippold SC, Goldman A, et al. Neurobehavioural functioning and magnetic resonance imaging in young boxers. J Neurosurg 1987;67:657-67

15. Jordan BD, Zimmerman RD. Magnetic resonance imaging in amateur boxers. Arch Neurol 1988;45:1207-08

16. Holzgraefe M, Lemme W, Funke W, et al. The significance of diagnostic imaging in acute and chronic brain damage in boxing: a prospective study in amateur boxing using magnetic resonance imaging (MRI). Int J Sports Med 1992;13:616-20

17. Adams JH, Mitchell DE, Graham DI, et al. Diffuse brain damage of the immediate type. Brain 1977;100:489-502

18. Tong KA, Ashwal S, Holshouser BA, et al. Hemorrhagic shearing lesions in children and adolescents with posttraumatic diffuse axonal injury: improved detection and initial results. Radiology 2003;227:332-39

19. Tong KA, Ashwal S, Holshouser BA, et al. Diffuse axonal injury in children: clinical correlation with hemorrhagic lesions. Ann Neurol 2004;56:36-50

20. Babikian T, Freier MC, Tong KA, et al. Susceptibility weighted imaging: neuropsychologic outcome and pediatric head injury. Pediatr Neurol 2005;33: 184-94 